\title{
Guest editorial: focused section on human-centered robotics
}

\author{
Santosh Devasia ${ }^{1} \cdot$ Chien Chern Cheah $^{2} \cdot$ Marcello Pellicciari $^{3} \cdot$ Margherita Peruzzini $^{4}$
}

Published online: 17 May 2018

(c) Springer Nature Singapore Pte Ltd. 2018

Human-centered robotics requires a fundamental shift from the traditional robot-in-a-cage model to robots interacting with people in an open environment with applications that include wearable robotics, social robots for home and entertainment, and collaborative manufacturing. Many efforts are underway to guarantee safety and improve performance when such robots operate in human environments, such as improved sensors and actuators and data-based methods for enhanced cognition. This generates numerous opportunities for innovation. At the same time there are substantial challenges before such robots become ubiquitous.

To disseminate current advances and identify challenges and opportunities, this "Focused Section on Human-Centered Robotics" of the International Journal of Intelligent Robotics and Applications (IJIRA) highlights several important research and technology achievements in: human-robot interface; action and trajectory planning; sensors; safety; and humans teaming with multiple robots. The Focused Section includes nine papers that represent a sample of current developments in human-centered robotics.

Intent and interface Human-centered robotics should have appropriate interfaces to assess human intent and facilitate

Santosh Devasia

devasia@uw.edu

Chien Chern Cheah

ecccheah@ntu.edu.sg

Marcello Pellicciari

marcello.pellicciari@unimore.it

Margherita Peruzzini

margherita.peruzzini@unimore.it

1 Mechanical Engineering Department, University of Washington, Seattle, USA

2 School of Electrical and Electronic Engineering, Nanyang Technological University, Singapore, Republic of Singapore

3 Department of Sciences and Methods for Engineering (DISMI), University of Modena and Reggio Emilia, Reggio Emilia, Italy

4 Department of Engineering "Enzo Ferrari”, University of Modena and Reggio Emilia, Modena, Italy human use of robotic systems. The first paper "Gaze and motion information fusion for human intention inference" by Ravichandar et al. presents an algorithm to estimate human intent (target location) based on human reaching actions. Additionally human gaze is used to improve the estimated intent and quantitative evaluations using multiple subjects are presented. The second article "Sequence-based manipulation of robotic arm control in brain machine interface" by Kilmarx et al. uses a new robotic Brain-Machine-Interface (BMI) platform using Electroencephalography (EEG) technology to control a six degree of freedom robotic arm, which can impact the use of such interfaces to control prosthesis. A key result is that the new approach provided good results with about ten minutes of training, which is substantially smaller than the typical days to weeks of training needed with standard BMI approaches that use sensorimotor rhythm to control robotic systems.

Motion planning The article "Robot action planning by online optimization in human-robot collaborative tasks" by Wang et al. addresses the issue of task cost evaluation along with online optimization, which can be used for planning of human-robot collaborative tasks such as manufacturing. Their experimental results indicate that the proposed algorithms can enable efficient human-robot collaborative assembly. The rich set of issues in motion planning even for a specific task is highlighted in the article "Momentumbased trajectory planning for lower-limb exoskeletons supporting sit-to-stand (STS) transitions" by Patil et al. The paper analyses factors that affect human center of mass trajectory and shows that different human movement velocity profiles during STS transitions require different control strategies of the center of mass. The researchers propose a model based on horizontal and vertical momentums that enables efficient planning of the center of mass trajectory for any STS transition velocity. Such efforts to improve STS can enhance independent living for seniors.

Sensing and safety Assessing the human mental and physical state, which in turn can be important for safety in human-centered robotics. The article "Development of a combined time-grequency technique for accurate extraction 
of pNN50 metric from noisy heart rate measurements" by Vaqueiro et al. addresses the measurement of a key metric associated with human mental workload. Such measurements could inform the robot about the degree to which "a human is overwhelmed and error-prone in decision making". The article "Automatic analysis of ultrasound shear-wave elastography in skeletal muscle without non-contractile tissue contamination" by Brown et al. develops a new ultrasound image processing methods to assess muscle activities. Such assessment could be used in clinical settings as a diagnostic tool for monitoring rehabilitation from neuromuscular disease and injury such as stroke and spinal cord injury. The safety of human-robot interaction can also be highly dependent on which part of the body the robot is interacting with and the pose of the body. The work "A design metric for safety assessment of industrial robot design suitable for power and force limited collaborative operation" by Vemula et al. aims to develop simulation tools to assess the safety of transient contact during human-robot collaboration.

Managing multiple robots The article "Motion synchronization for semi-autonomous robotic swarm with a passivityshort human operator" by Atman et al. investigates coordination between a human operator and a robotic swarm where the goal is to guarantee human-enabled motion synchronization to desired position/velocity references. The proposed architecture is demonstrated through simulation studies and with an experimental test-bed. As mentioned in the article, such swarm control can be important for "environmental monitoring, infrastructure support or exploration due to its scalability and robustness against robot failures". The article "Collaborative manipulation with multiple dual-arm robots under human guidance" by Peng et al. develops a method for using multiple robots to collaboratively manipulate an object when the load and size cannot be managed by a single robot. The article uses contact force sensing and infers the human intent to enable collaborative manipulation of the object without loss of contact. Inertial forces are estimated and compensated using feedforward and experimental results are provided to illustrate the efficacy and limitations of the proposed scheme.

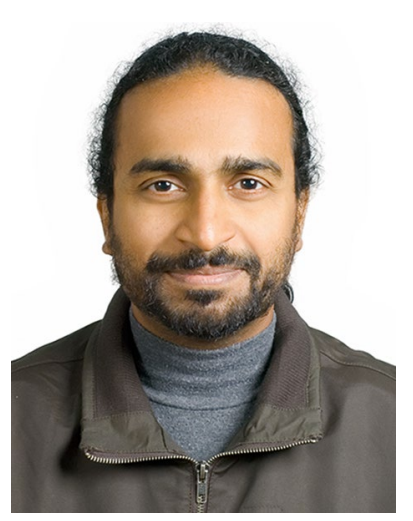

Santosh Devasia is a professor of Mechanical Engineering at the U. of Washington, Seattle. He got his B. Tech. (Hons) degree from the Indian Institute of Technology, Kharagpur (India) and the M.S. and Ph.D. Degrees from the University of California at Santa Barbara. He is a Fellow of ASME and a senior member of IEEE. He served on the Management Committee for the IEEE/ASME Transactions on Mechatronics, is on the
Executive Committee of the ASME Dynamic Systems and Control Division (DSCD), was an Associate Editor for the IEEE Trans. on Control Systems Technology and the ASME Journal of Dynamic Systems Measurement and Control, and is the General Chair of the 2020 American Control Conference. He serves as the Director of the Boeing Advanced Research Center https://depts.washington.edu/barc at the University of Washington, which is focused on Human-Robot shared operations for Confined Space Manufacturing, that includes research on precision series-elastic actuators. His current research interests include human-robot interaction in manufacturing (aerospace assembly), iterative learning and distributed systems.

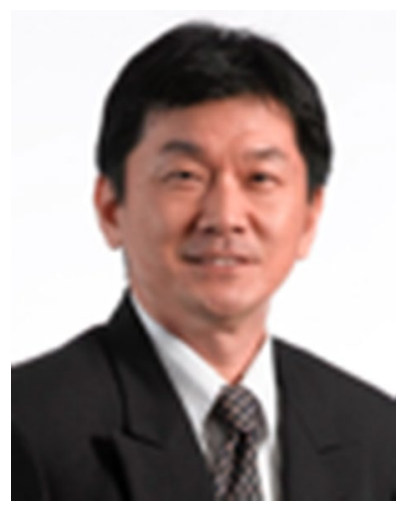

Chien Chern Cheah received his B.E. degree in Electrical Engineering from National University of Singapore in 1990, M.E. and Ph.D. degrees in Electrical Engineering, both from Nanyang Technological University, Singapore, in 1993 and 1996, respectively. He is currently an associate professor in Nanyang Technological University. He serves as an associate editor for Automatica and an editorial board member for Robotics and Biomimetics. He has served as an associate editor for IEEE

Transactions on Robotics and Asian Journal of Control. He was the program chair of the International Conference on Control, Automation, Robotics and Vision (ICARCV) in 2006 and 2012, and the program co-chair of IEEE International Conference on Robotics and Automation (ICRA) 2017. His current research interests include adaptive and learning control, human-robot interaction, and micro-manipulation.

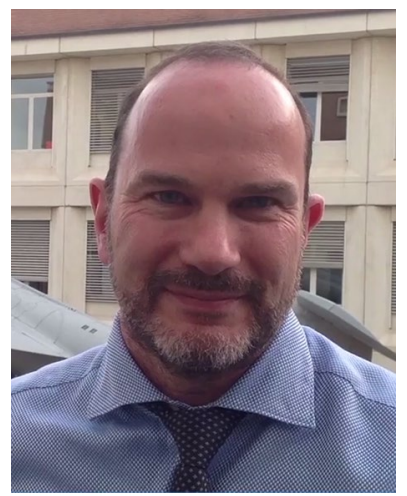

Marcello Pellicciari received the B.Sc. degree in engineering in 1997 and the PhD Degree in 2001 at the University of Modena and Reggio Emilia, Italy, where he is currently serving as full professor in Engineering Design Methods and Tools. He is Chair of the Engineering Design course at the Department of Engineering "Enzo Ferrari" and member of the Board of SIRI, the Italian Robotics and Automation Society, as well as member of the IEEE technical committee on sustainable production automation. His current research interests are integrated design and virtual prototyping of mechatronic systems, industrial robotics and sustainable manufacturing. He was the General Chair of the 27th International Conference on Flexible Automation and Intelligent Manufacturing (FAIM2017). 


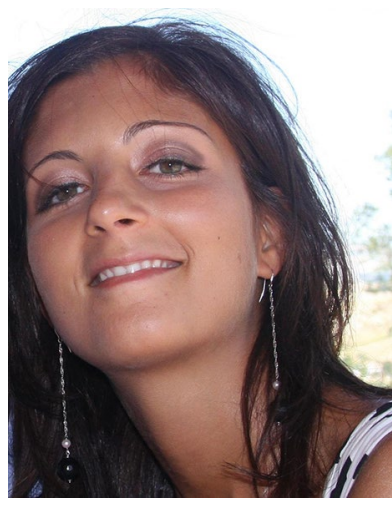

Margherita Peruzzini is an Associate Professor at the Department of Engineering "Enzo Ferrari", University of Modena and Reggio Emilia, and works in the research area of Methods and Tools for Industrial Engineering. She graduated in Mechanical Engineering in 2007 and she received her $\mathrm{Ph} . \mathrm{D}$. in Mechanical Engineering and Engineering Management in 2010. She is part of LAPIS (Laboratory of integrated Design and Simulation) and INTERMECH MO.RE, an inter-department Lab of the University of Modena and Reggio Emilia that works on advance mechanics for industrial applications. She coordinates the Vip LAB (Virtual
Prototyping Lab) of the Modena Technopole. Her topics of research are: Virtual prototyping and industrial applications, Human-Centred Design, Human-Computer Interaction and Human-Machine Interfaces, Collaborative Virtual Environments, Co-design, Product-Service Systems. She also participates in industrial research projects at national and European level. She is a member of the Design society and other scientific communities, and she is author of more than 120 international publications on journals and conference proceedings. She has served as Guest Editors for different international journals from Elsevier, Inderscience, Taylor\&Francis and WorldScientific, and has been a reviewer of more than 20 different international journals. She was the Program Chair of several conferences (FAIM2017, TE2017, TE2016) and she is the Conference Chair of TE2018 conference (www.te2018. com). 